\title{
Granzyme B triggers a prolonged pressure to die in Bcl-2 overexpressing cells, defining a window of opportunity for effective treatment with ABT-737
}

\author{
VR Sutton ${ }^{1,2,11}$, K Sedelies ${ }^{1,2,11}$, G Dewson ${ }^{3,11}$, ME Christensen ${ }^{4}$, PI Bird ${ }^{5}$, RW Johnstone, ${ }^{6,7,8,2}$, RM Kluck ${ }^{9,11}$, JA Trapani ${ }^{1,7,8,2,12}$ \\ and NJ Waterhouse ${ }^{\star, 4,10,12}$
}

Overexpression of $\mathrm{Bcl}-2$ contributes to resistance of cancer cells to human cytotoxic lymphocytes (CL) by blocking granzyme $B$ (GraB)-induced mitochondrial outer membrane permeabilization (MOMP). Drugs that neutralise Bcl-2 (e.g., ABT-737) may therefore be effective adjuvants for immunotherapeutic strategies that use $\mathrm{CL}$ to kill cancer cells. Consistent with this we found that ABT-737 effectively restored MOMP in Bcl-2 overexpressing cells treated with GraB or natural killer cells. This effect was observed even if ABT-737 was added up to $16 \mathrm{~h}$ after GraB, after which the cells reset their resistant phenotype. Sensitivity to ABT-737 required initial cleavage of Bid by GraB (gctBid) but did not require ongoing GraB activity once Bid had been cleaved. This gctBid remained detectable in cells that were sensitive to ABT-737, but Bax and Bak were only activated if ABT-737 was added to the cells. These studies demonstrate that GraB generates a prolonged pro-apoptotic signal that must remain active for ABT-737 to be effective. The duration of this signal is determined by the longevity of gctBid but not activation of Bax or Bak. This defines a therapeutic window in which ABT-737 and CL synergise to cause maximum death of cancer cells that are resistant to either treatment alone, which will be essential in defining optimum treatment regimens.

Cell Death and Disease (2012) 3, e344; doi:10.1038/cddis.2012.73; published online 5 July 2012

Subject Category: Cancer

Cytotoxic lymphocytes $(C L)$, which include natural killer cells (NK) and cytotoxic T lymphocytes (CTL), protect the body from cancer or infection and also contribute to the anti-cancer immune response that can accompany chemotherapy. ${ }^{1-3}$ $\mathrm{CL}$ primarily kill their targets by granule-induced death, a process that involves perforin (Pfp)-mediated delivery of granzyme B (GraB) to the target cell where it induces apoptosis. ${ }^{4,5}$ Understanding why some cancers are resistant to granule-induced death should lead to strategies that restore $\mathrm{CL}$-induced death when this process has failed.

We and others have shown that human GraB preferentially activates the mitochondrial pathway to apoptosis by cleaving the $\mathrm{Bcl}-2$ family member Bid. $^{6-10}$ Truncated Bid (tBid) promotes mitochondrial outer membrane permeabilization (MOMP), which liberates cytochrome $c$ (cyt $c$ ) to promote caspase activation. ${ }^{11,12}$ This pathway is blocked by prosurvival $\mathrm{Bcl}-2$ family members that are commonly elevated in cancer. ${ }^{12,13}$ GraB can also initiate apoptosis by directly activating caspases. This pathway can be negatively regulated by X-linked inhibitor of apoptosis protein (XIAP), which is also elevated in many cancers. ${ }^{14,15}$ However, MOMP also releases second mitochondrial activator of caspases (Smac), which can neutralise XIAP. ${ }^{15}$ GraB can therefore activate two independent pathways to caspase activation that bypass antiapoptotic proteins commonly elevated in cancer. However, blocking both pathways by overexpression of $\mathrm{Bcl}-2$ together with XIAP can prevent granule-induced cell death by human NK (14) and these target cells continue to proliferate. Overexpression of these proteins may therefore be one way that cancer cells avoid CL-induced death and why some patients fail to respond to anti-cancer immunotherapy. These studies also suggest that restoring the mitochondrial pathway should be sufficient to re-sensitise these cancer cells to CL-induced death.

\footnotetext{
${ }^{1}$ Cancer Cell Death Laboratory, Cancer Immunology Program, Peter MacCallum Cancer Centre, Locked Bag 1, A'Beckett Street, Melbourne, Victoria 8006, Australia; ${ }^{2}$ Sir Peter MacCallum Department of Oncology, The University of Melbourne, Melbourne, Victoria 3052, Australia; ${ }^{3}$ Cell Signalling and Cell Death Division, The Walter and Eliza Hall Institute of Medical Research, 1G Royal Parade, Parkville, Victoria 3050, Australia; ${ }^{4}$ Apoptosis and Cytotoxicity Laboratory, Mater Medical Research Institute, Aubigny Place, Raymond Terrace, South Brisbane, Queensland 4101, Australia; ${ }^{5}$ Department of Biochemistry and Molecular Biology, Monash University, Melbourne, Victoria 3800, Australia; ${ }^{6}$ Gene Regulation Laboratory, Cancer Therapeutics Program, Peter MacCallum Cancer Centre, Locked Bag 1, A'Beckett Street, Melbourne, Victoria 8006, Australia; ${ }^{7}$ Department of Microbiology and Immunology, University of Melbourne, Parkville, Victoria 3052, Australia; ${ }^{8}$ Victorian Comprehensive Cancer Centre, Parkville, Victoria 3052, Australia; ${ }^{9}$ Molecular Genetics of Cancer Division, The Walter and Eliza Hall Institute of Medical Research, 1 G Royal Parade, Parkville, Victoria 3050, Australia; ${ }^{10}$ Department of Medicine, University of Queensland, St Lucia, Queensland, Australia and ${ }^{11}$ Department of Medical Biology, The University of Melbourne, Parkville, Victoria 3010, Australia

${ }^{*}$ Corresponding author: NJ Waterhouse, Apoptosis and Cytotoxicity Laboratory, Mater Medical Research Institute, Aubigny Place, Raymond Terrace, South Brisbane, Queensland 4101, Australia. Tel: +61 73163 2584; Fax: +61 73163 2550; E-mail: nwaterhouse@mmri.mater.org.au

${ }^{12}$ These authors contributed equally to this work.

Keywords: granzyme; lymphocyte; Bcl-2; apoptosis; mitochondria; ABT-737

Abbreviations: Cc, Caspase-cleaved; C20, Compound 20; cyt c, cytochrome c; CL, cytotoxic lymphocytes; CTL, cytotoxic T lymphocytes; gctBid, GraB-cleaved Bid; GraB, human granzyme B; MOMP, mitochondrial outer membrane permeabilization; NK, natural killer cells; Pfp, perforin

Received 19.12.11; revised 23.4.12; accepted 02.5.12; Edited by P Salomoni
} 
Consistent with this, the small molecule antagonist of Bcl-2, ABT-737, has been shown to re-sensitise some cancer cells to CTL-induced death, ${ }^{16}$ suggesting that it may have adjuvant activity for cell-based cancer immunotherapy. It is not clear how ABT-737 re-sensitises cancer cells to CL or GraB or how to use ABT-737 most appropriately in future clinical trials that combine drug- and immune-based therapies. We have therefore investigated the synergy between ABT-737 and GraB, and show that (1) GraB generates a persistent pro-apoptotic signal to die in cancer cells that overexpress $\mathrm{Bcl}-2$ that can be released by ABT-737 even if GraB activity is subsequently blocked; (2) this signal is transduced by tBid but is blocked by Bcl-2 upstream of Bax and Bak, and neutralising $\mathrm{Bcl}-2$ with $\mathrm{ABT}-737$ then results in rapid activation of Bax and Bak and MOMP; and (3) the pro-apoptotic signal generated by GraB and blocked by $\mathrm{Bcl}-2$ is surprisingly long lived and MOMP can be triggered by adding ABT-737 as long as $20 \mathrm{~h}$ after Pfp/GraB. This defines a 'window of opportunity' when ABT-737 and GraB can jointly kill cancer cells that overexpress Bcl-2, which will be important for defining treatment regimens to use $A B T-737$ most effectively in combination with immunotherapy.

\section{Results}

GraB generates a persistent apoptotic signal that is rapidly released by $\mathrm{ABT}-737$. As MOMP is the critical event in apoptosis that is regulated by $\mathrm{Bcl}-2$ family members ${ }^{12,15,17,18}$ we first used immunofluorescence staining for cyt $c$ to investigate whether ABT-737 could restore MOMP in HeLa-Bcl-2 cells treated with Pfp/GraB or NK. HeLa-Bcl-2 cells treated with NK cells or Pfp/GraB alone showed punctate staining typical of intact mitochondria. However, staining became diffuse in HeLa-Bcl-2 cells treated with GraB- or NK in the presence of ABT-737, indicating that cyt $c$ release had occurred (Figures $1 \mathrm{a}$ and b). Importantly, this effect was owing to restoration of the mitochondrial pathway, because the caspase-inhibitor zVAD-fmk was added in these assays to prevent any contribution of caspases directly activated by GraB. Consequently, ABT-737 did not restore apoptosis per se in these assays. To formally demonstrate that ABT-737 could restore GraBinduced apoptosis in the HeLa-Bcl-2 cells we used conditions that activate the mitochondrial pathway, but do not activate caspases directly. ${ }^{7,14,18}$ As expected, Bcl-2 overexpressing cells were resistant to GraB-induced apoptosis under these conditions but apoptosis was restored by ABT-737 as determined by annexin $\mathrm{V}$ binding (Figure 1c) or release of ${ }^{51} \mathrm{Cr}$ from the targets cells (Figure $1 \mathrm{~d}$ ). Death was restored using low concentrations of ABT-737 (Figure 1e), but was not restored by an inactive enantiomer of $A B T-737$ that cannot neutralise Bcl-2 (Figure 1c) or if the cells were pre-treated with compound 20 (C20), which specifically blocks the activity of human GraB (14) (Figure 1d); thereby showing strong synergy between both GraB and ABT-737 to kill the $\mathrm{Bcl}-2$ overexpressing cells.

Interestingly, ABT-737 was fully effective in restoring GraBinduced death of HeLa-Bcl-2 cells in these assays even if it was added $1 \mathrm{~h}$ after $\mathrm{Pfp} / \mathrm{GraB}$ as shown by equivalent ${ }^{51} \mathrm{Cr}$ release (Figure 1f), or if GraB activity was blocked by adding C20 after $30 \mathrm{~min}(t=30 \mathrm{~min})$ followed by ABT-737 after $1 \mathrm{~h}$ (Supplementary Figure 1). This suggested that GraB rapidly generates a pro-apoptotic signal in Bcl-2 overexpressing cells (within $30 \mathrm{~min}$ ) that is sustained for at least another $30 \mathrm{~min}$ even if GraB activity is blocked. This prompted us to investigate the nature of this pro-apoptotic signal. To investigate this we first used time-lapse microscopy to follow HeLa-Bcl-2 cells pre-treated with $\mathrm{Pfp} / \mathrm{GraB}$ for $1 \mathrm{~h}$ followed by addition of ABT-737. Cyt $c$ was located in the mitochondria of the Pfp/GraB-treated HeLa-Bcl-2 cells but rapidly translocated to the cytoplasm almost immediately after ABT-737 was added (within $15 \mathrm{~min}$ ) and the cells subsequently showed classic signs of apoptosis including rounding and blebbing (Supplementary Movie 1). Similar experiments using flow cytometry to quantify cyt $c$ release also revealed that ABT-737 triggered maximum cyt $c$ release within 15 min in HeLa-Bcl-2 cells that had been pre-treated with $\mathrm{Pfp} / \mathrm{GraB}$ for $1 \mathrm{~h}$ (Figure 2a). Immunoblot analysis also showed that although caspases were partially cleaved in HeLa-Bcl-2 cells treated with $\mathrm{Pfp} / \mathrm{GraB}$ after $1 \mathrm{~h}$, consistent with previous studies, ${ }^{15}$ caspase-3, -7 and -9 were fully processed to their active forms within 20 min of adding ABT-737 (Supplementary Figure 2, long arrows). This confirmed MOMP was required for full caspase activation and that ABT-737 treatment replicated the death observed in cells expressing endogenous levels of $\mathrm{Bcl}-2$. These experiments demonstrated that $\mathrm{ABT}-737$ rapidly de-represses the anti-apoptotic effect of Bcl-2 to trigger cyt $c$ release and caspase activation in a near-simultaneous manner in cells that had been pre-treated with Pfp/GraB.

The rapid release of cyt $c$ by ABT-737 in these assays suggested that the mitochondria were 'primed' to initiate MOMP in these cells once Bcl-2 had been neutralised, and raised some important questions. The first was how long do the mitochondria of $\mathrm{Bcl}-2$ overexpressing cells remain primed to release cyt $c$ ? This is important because NK disengage from their targets after they deliver GraB. Sensitivity of their targets to ABT-737 may therefore be limited. How long the GraB-treated cells remain sensitive to ABT-737 may therefore

\footnotetext{
Figure 1 ABT-737 restores cell death of HeLa-Bcl-2 cells treated with human NK cells or GraB. HeLa-Bcl-2 cells were treated with (a) human NK cells (stimulated with $25 \mathrm{U}$ IL-2 for 4 days), or (b) Pfp ( $1 \mathrm{nM})$ and GraB $(25 \mathrm{nM})$ in the presence of zVAD-fmk (100 $\mu \mathrm{M})$. ABT-737 (500 nM) was added at $t=0$ and cells were fixed after $4 \mathrm{~h}$. Images of morphology (DIC) and cyt c location (immunofluorescence) were taken using an Olympus CellR fluorescence microscope with a $\times 40$ oil-immersion lens. Arrows indicate cells that have released cyt $c$ and asterix are cells that have not. (c) HeLa-Bcl-2 cells were treated with Pfp $(1 \mathrm{nM}) / \mathrm{GraB}(25 \mathrm{nM})$ in the presence or absence of ABT-737 (500 nM) or Enantiomer $(500 \mathrm{nM})$. Cell death was determined by Annexin V binding. Data are the average \pm S.E.M. for three independent experiments. (d) HeLa-Bcl-2 cells were treated with Pfp $(1 \mathrm{nM}) / \mathrm{GraB}(25 \mathrm{nM})$ in the presence or absence of ABT-737 $(500 \mathrm{nM}) \pm \mathrm{GraB}$ inhibitor $(\mathrm{C} 20 ; 10 \mu \mathrm{M})$ for $4 \mathrm{~h}$. Cell death was determined by release of ${ }^{51} \mathrm{Cr}$ that had been pre-loaded into the target cells. Data are the average \pm S.E.M. for three independent experiments. (e) HeLa-Bcl-2 cells were pre-labelled with ${ }^{51} \mathrm{Cr}$ and incubated with $\operatorname{Pfp}(1 \mathrm{nM}) / \mathrm{GraB}(12.5 \mathrm{nM})$ together with the concentration of ABT-737 indicated. Media containing $10 \% \mathrm{FCS}$ was added at $1 \mathrm{~h}$. Specific ${ }^{51} \mathrm{Cr}$ released from the target cells was measured at $4 \mathrm{~h}$ as an indication of cell death. Data are the average \pm S.E.M. of five experiments. (f) ${ }^{51} \mathrm{Cr}$ release from HeLa-Bcl-2 cells treated with Pfp (1 nM)/GraB $(12.5 \mathrm{nM})$ for $4 \mathrm{~h}$. ABT-737 (500 nM) was either omitted (No. ABT-737) or added to the HeLa-Bcl-2 cells as indicated either at 0 min (ABT-737, $t=0)$ or after 60 min (ABT-737, $t=60)$
} 
a
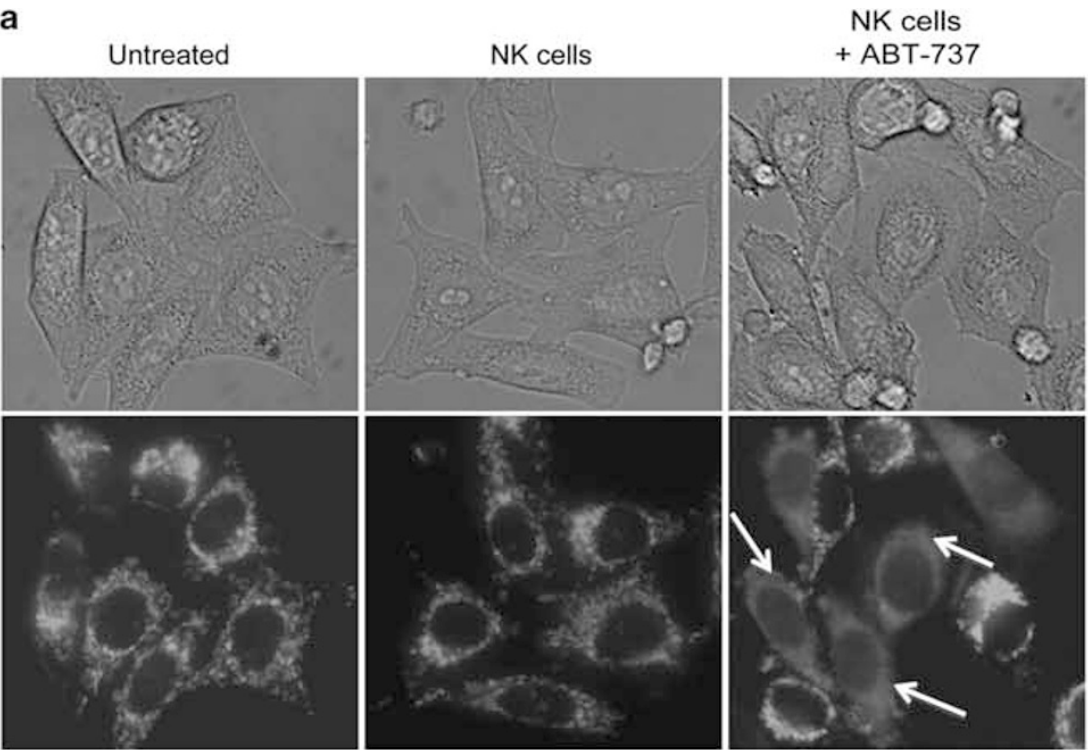

b

ABT-737

Pfp

Pfp/GraB

$\mathrm{Pfp} / \mathrm{GraB}$
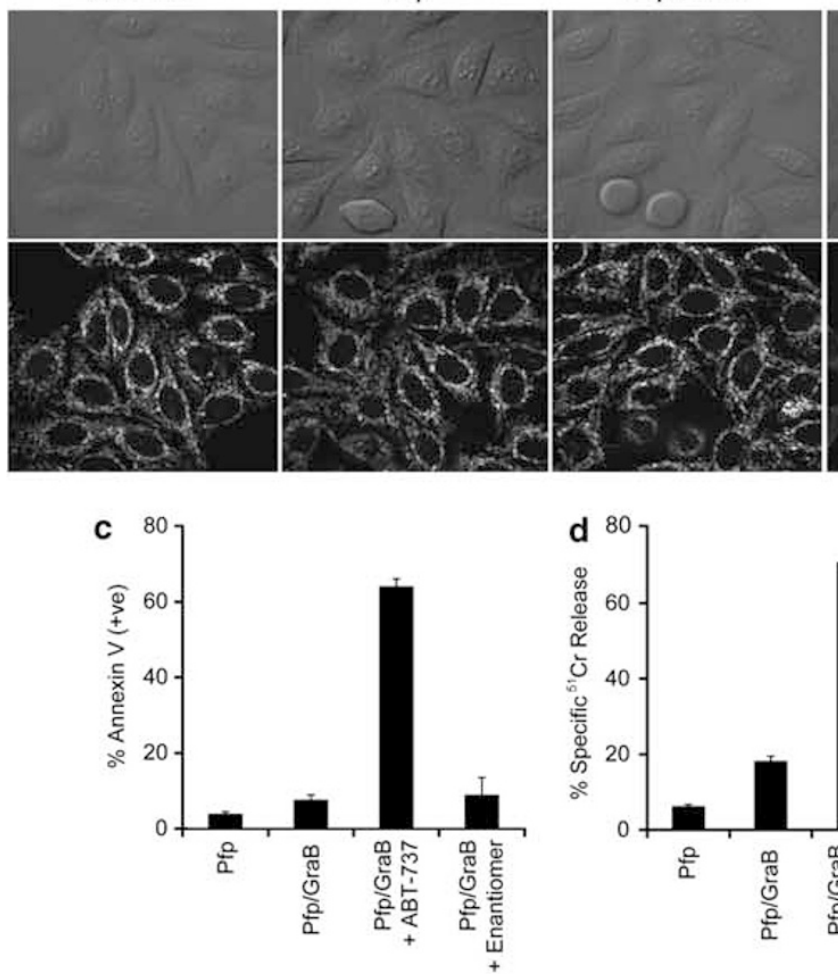

+ ABT-737
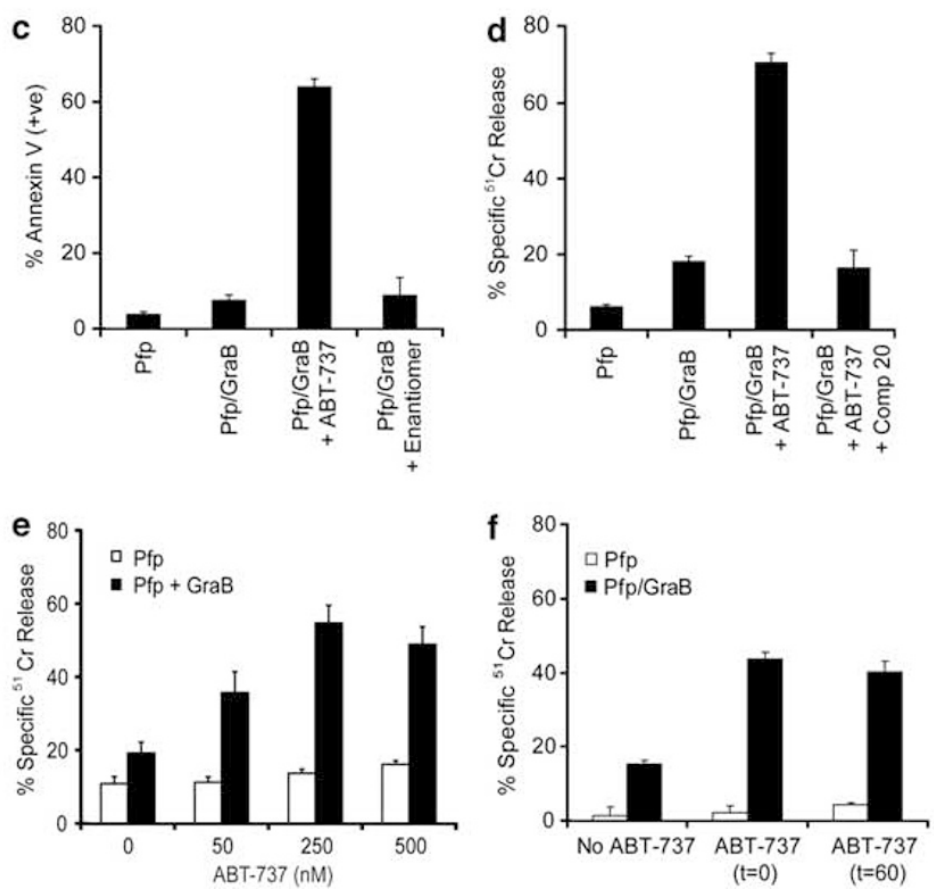
influence the design of treatment regimens using ABT-737 with immunotherapy. To investigate this, we delayed addition of ABT-737 to Pfp/GraB-treated HeLa-Bcl-2 cells for various times up to $24 \mathrm{~h}$. At each time-point, cyt $c$ release was assayed 15 min after adding ABT-737 (Figure 2b), sufficient
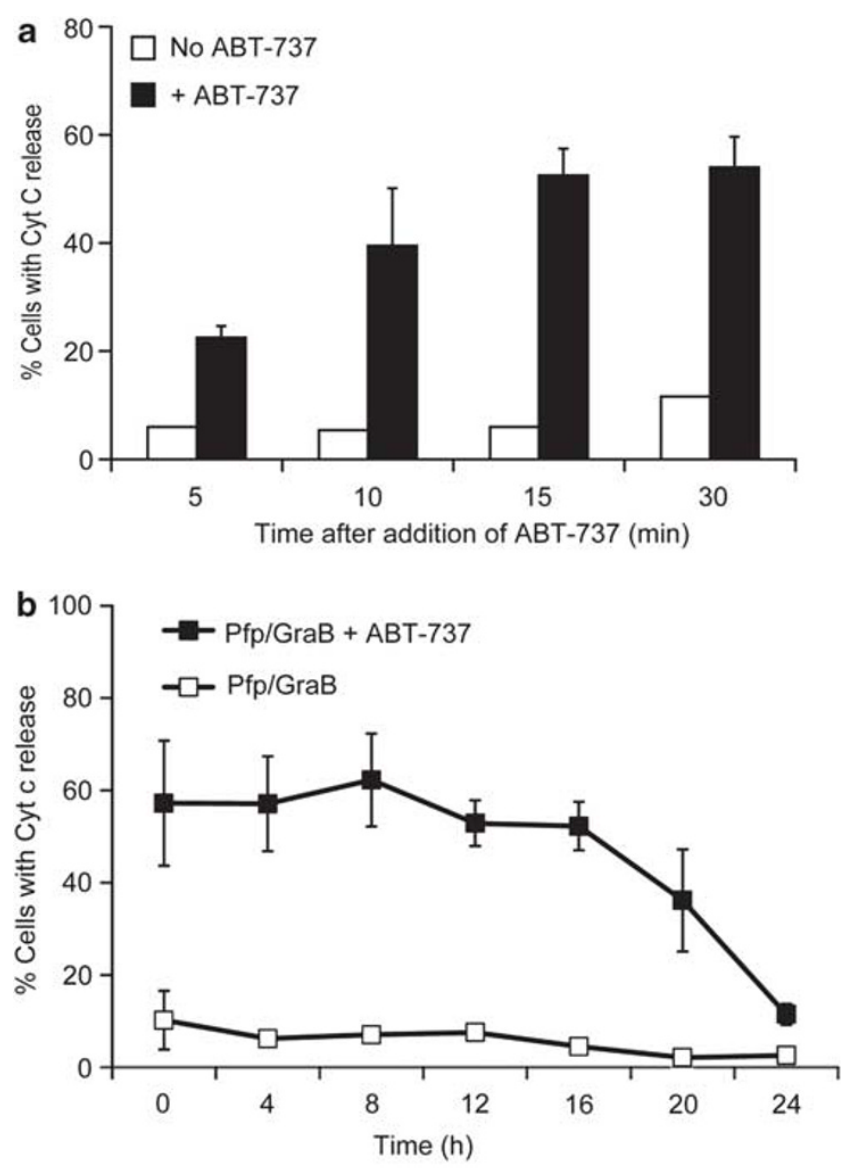

C

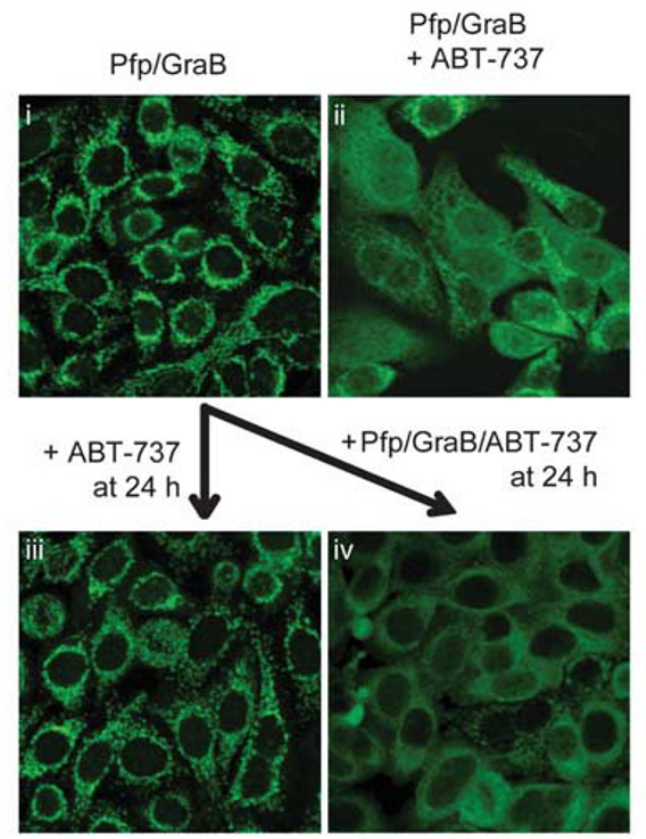

time for ABT-737 to trigger maximal cyt $c$ release in mitochondria primed to undergo MOMP (Figure 2a). The majority of cells released cyt $c$ even when ABT-737 was added $16 \mathrm{~h}$ after Pfp/GraB. Approximately half were still responsive at $20 \mathrm{~h}$ but the death signal was finally lost by $24 \mathrm{~h}$. The surviving cells were still sensitive to further treatment with Pfp/GraB and ABT-737 after $24 \mathrm{~h}$ (Figure 2c). Overall, these studies revealed that GraB generates a long but finite proapoptotic signal that defines a 'window of opportunity' during which the Bcl-2 overexpressing cells are sensitive to ABT-737 and that these cells are likely to respond to multiple rounds of combined therapy with NK and ABT-737.

Bcl-2 blocks the pro-apoptotic signal initiated by GraB by binding to tBid upstream of Bax translocation and Bax/Bak activation. The second question raised by our observations was: what is the nature of priming that persists in GraB-treated HeLa-Bcl-2 cells to render them sensitive to $\mathrm{ABT}-737$, and is persistent proteolytic activity by GraB required to sustain this pressure throughout the 'window of opportunity'? The molecular mechanism of GraB-induced MOMP involves direct cleavage of Bid at Asp75 and migration of tBid to the mitochondria where it activates the multimeric pro-apoptotic Bcl-2 family members (Bax or Bak) to cause MOMP. ${ }^{15,19-22}$ Consistent with this we have previously demonstrated that Bid cleavage occurs upstream of $\mathrm{Bcl}-2$ during GraB-induced death and that murine embryonic fibroblasts from both $\mathrm{Bid}^{-/-}$and $\mathrm{Bax}^{-/-} \mathrm{Bak}^{-/-}$ mice were resistant to GraB-induced death ${ }^{18}$ (and data not shown). Bcl-2 has been shown to bind and block the active form of all three pro-apoptotic proteins via different mechanisms depending on the strength and nature of the stimulus applied. ${ }^{22-27}$ We therefore investigated the role of Bid, Bax and Bak in maintaining the pressure to die in $\mathrm{Bcl}-2$ overexpressing cells.

As $\mathrm{Bcl}-2$ overexpressing cells were primed to undergo MOMP once ABT-737 was added at $1 \mathrm{~h}$ (Figure 1f, Figure 2), the pro-apoptotic signal must be active in these cells at this time. We therefore tested whether Bid, Bax or Bak were converted to their active conformation within $1 \mathrm{~h}$ of treatment with GraB. As expected, control HeLa cells treated with Pfp/ GraB generated a large amount of tBid (GraB-cleaved (gc) tBid), which was largely localised in the mitochondrial pellet, and also produced smaller amounts of a slower-migrating form of tBid cleaved directly by caspases (caspase-cleaved

Figure 2 ABT-737 can trigger rapid and maximal cyt $c$ release in HeLa-Bcl-2 cells up to $16 \mathrm{~h}$ after $\mathrm{Pfp} / \mathrm{GraB}$ has delivered the apoptosis-inducing signal. (a) HeLa-Bcl-2 cells were pre-treated with Pfp ( $1 \mathrm{nM}) / \mathrm{GraB}(25 \mathrm{nM})$ for $1 \mathrm{~h}$. ABT-737 $(500 \mathrm{nM})$ was then added (designated as $t=0)$ and cells were assayed for cyt $c$ release by flow cytometry at 5, 10, 15 and 30 min after adding ABT-737. (b) HeLaBcl-2 cells were treated with Pfp $(1 \mathrm{nM}) / \mathrm{GraB}(25 \mathrm{nM})$ and zVAD-fmk $(100 \mu \mathrm{M})$. ABT-737 (500 nM) was added at the time points indicated. Cells were harvested after $15 \mathrm{~min}$ and analysed for cyt $c$ release by flow cytometry. (c) HeLa-Bcl-2 cells were treated with Pfp $(1 \mathrm{nM}) / \mathrm{GraB}(25 \mathrm{nM})$ and zVAD-fmk $(100 \mu \mathrm{M})$ alone (Panel i) or together with ABT-737 $(250 \mathrm{nM})$ and images of cyt $c$-GFP were obtained at $4 \mathrm{~h}$. Similarly, cells were treated for $24 \mathrm{~h}$ then either ABT-737 alone (Panel iii) or ABT-737 together with Pfp ( $1 \mathrm{nM}) / \mathrm{GraB}(25 \mathrm{nM})$ and zVAD-fmk $(100 \mu \mathrm{M})$ (Panel iv) was added. Images of cyt $c$-GFP were obtained at $28 \mathrm{~h}$ using a Leica-SP5 confocal microscope (North Ryde, NSW, Australia) 

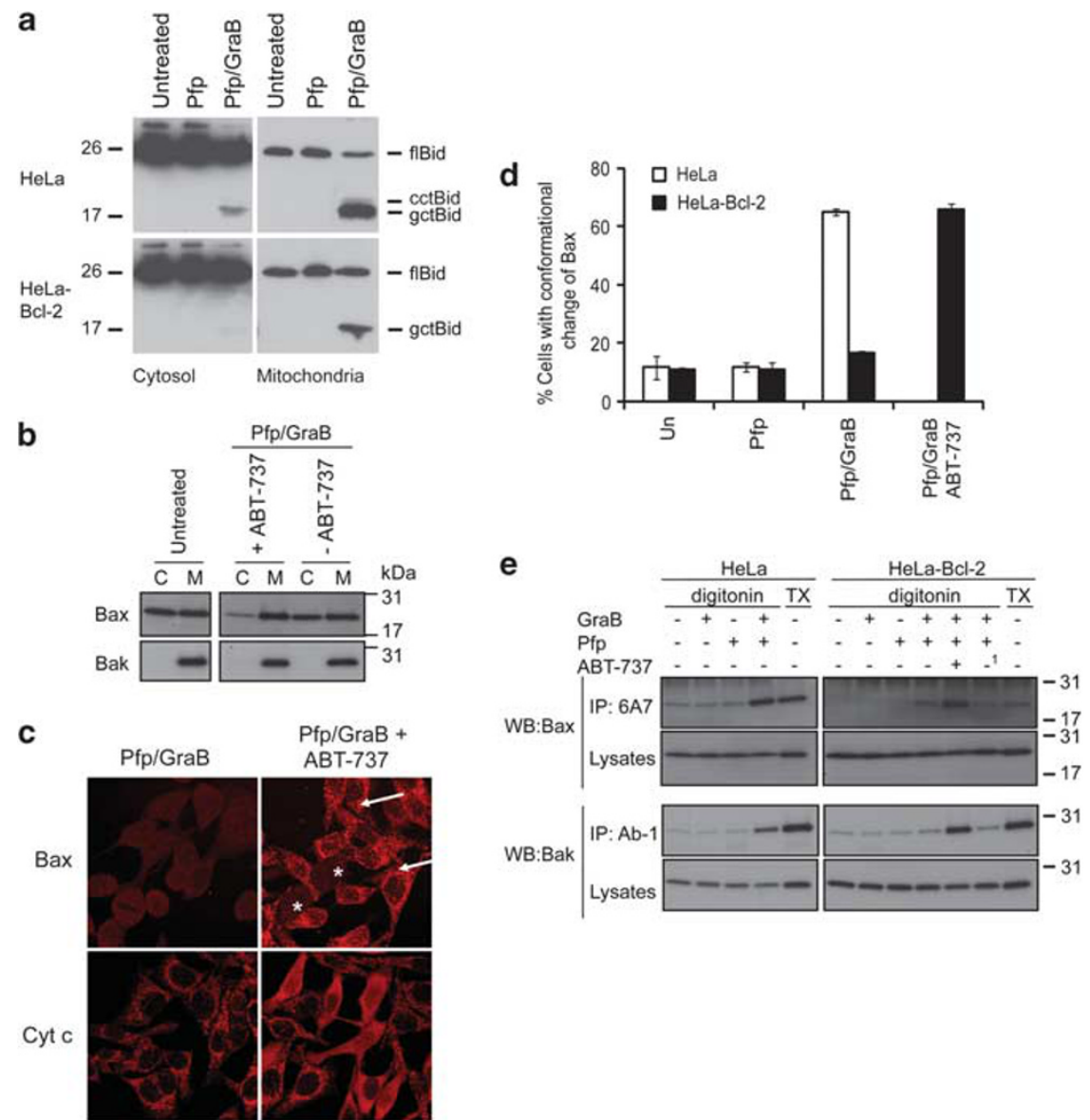

Figure 3 Bid cleavage by GraB and translocation of gctBid to the mitochondria is not blocked by Bcl-2 but Bax translocation and Bax/Bak activation are inhibited until after ABT-737 treatment. (a) Cells were treated with Pfp/GraB for $1 \mathrm{~h}$. Cytosolic and membrane fractions were harvested and full length (fl) and tBid were detected by western blot analysis. Both GraB-cleaved tBid (gctBid) and caspase-cleaved tBid (cctBid) were detected in treated HeLa cells but only gctBid was detected in the HeLa-Bcl-2 cells. (b) HeLa-Bcl-2 cells were treated with Pfp/GraB for $1 \mathrm{~h}, \mathrm{ABT}-737$ was added for $30 \mathrm{~min}$ as indicated, before fractionation into cytosolic (C) and membrane (M) fractions and immunoblotting for Bax and Bak. Data are representative of three independent experiments. (c) HeLa-Bcl-2 cells were treated with Pfp ( $1 \mathrm{nM}) / \mathrm{GraB}(25 \mathrm{nM})$ and zVAD-fmk $(100 \mu \mathrm{M})$. ABT-737 $(500 \mathrm{nM})$ was added to the cells indicated after $1 \mathrm{~h}$ and incubated for a further 30 min before they were fixed and stained for Bax and cyt $c$. Images were obtained using an Olympus FV1000 confocal microscope. Cells marked with an arrow indicate cells with active Bax and those with an asterix indicate cells without active Bax. (d) HeLa and HeLa-Bcl-2 cells were treated with Pfp $(1 \mathrm{nM}) / \mathrm{GraB}(25 \mathrm{nM})$ for $1 \mathrm{~h}$. ABT-737 (500 nM) was added for a further 30 min. Flow cytometry was used to detect Bax activation using an antibody specific for the conformational-active form of Bax (clone 6A7). Data are average \pm S.D. of four independent experiments. (e) Conformation change of Bax (upper panels) and Bak (lower panels) was determined by immunoprecipitation with conformation-specific antibodies against Bax (6A7) or Bak (Ab-1) (as described in the Materials and Methods). Lysis in Triton X-100 (TX) was used as a positive control for conformational change of Bax and Bak. ${ }^{1}$ Cells were incubated for 30 min in the absence of ABT-737 after Pfp/GraB treatment (for $1 \mathrm{~h}$ ). The experiment was performed twice

(cc) tBid) (Figure 3a). GctBid was also generated and was localised to the mitochondrial fraction of HeLa-Bcl-2 cells treated with $\mathrm{Pfp} / \mathrm{GraB}$, but cctBid was not observed as MOMP had not occurred. The presence of GraB-cleaved Bid (gctBid) at mitochondria in HeLa-Bcl-2 cells showed that the death signal had reached mitochondria in the absence of ABT-737 and gctBid accumulation at the mitochondria also provided a mechanism through which ABT-737 might potentially trigger rapid MOMP, even if GraB activity had ceased some time earlier.

We next examined whether Bcl-2 protects cells by sequestering gctBid or Bax/Bak that had been activated by gctBid, and whether ABT-737 liberated gctBid and/or activated $\mathrm{Bax} / \mathrm{Bak}$ from $\mathrm{Bcl}-2$ to induce MOMP. Bax is generally located in the cytoplasm and translocates to mitochondria to cause MOMP, whereas Bak is constitutively located at the mitochondria of healthy cells. Although the mechanism by which Bax and Bak promote MOMP remains to be fully elucidated, both molecules change conformation before oligomerising in the mitochondrial outer membrane. We therefore investigated the cellular location and conformational change of Bax and Bak in HeLa-Bcl-2 cells treated with $\mathrm{Pfp} / \mathrm{GraB}$ in the presence and absence of ABT-737. As expected, Bak was located exclusively in the mitochondrial fractions of treated and untreated cells (Figure $3 b$ ). Bax was also predominantly in the mitochondrial fraction of cells after treatment with Pfp/GraB and ABT-737 (Figure 3b).

The conformational change in Bax exposes its $\mathrm{N}$-terminus, which can then be detected with an antibody that binds specifically in this region (clone 6A7). Using flow cytometry, $>60 \%$ of $\mathrm{HeLa}$ cells treated with $\mathrm{Pfp} / \mathrm{GraB}$ alone were positive for the $6 \mathrm{~A} 7$ epitope as were a similar proportion of HeLa-Bcl-2 cells treated with Pfp/GraB and ABT-737; background $6 \mathrm{~A} 7$ binding occurred in the HeLa-Bcl-2 cells 
treated with Pfp/GraB in the absence of ABT-737 (Figure 3c). This was confirmed by immunocytochemistry, which showed punctate Bax staining (cells with arrows) in HeLa-Bcl-2 cells treated with Pfp/GraB and ABT-737 indicating mitochondrial localisation, which contrasted with the faint diffuse staining when these cells were treated with $\mathrm{Pfp} / \mathrm{GraB}$ alone (Figure 3d). The converse was observed for cyt $c$, located predominantly in mitochondria of $\mathrm{HeLa}-\mathrm{Bcl}-2$ cells treated with $\mathrm{Pfp} / \mathrm{GraB}$ (punctate staining), and only released into the cytoplasm when ABT-737 was added (diffuse staining). Finally, immunoprecipitation with $6 A 7$ showed activated Bax in HeLa-Bcl-2 cells treated with Pfp/GraB after ABT-737 addition but only background levels before (Figure $3 e$ ). Thus, Bax only became enriched at the mitochondria and acquired an active conformation if Bcl-2 was neutralised by ABT-737. With respect to Bak, immunoprecipitation with $A b-1$ antibody showed Bak was activated in Pfp/GraB-treated HeLa cells or HeLa-Bcl-2 cells treated with Pfp/GraB and ABT-737, but not activated in HeLa-Bcl-2 cells treated with $\mathrm{Pfp} / \mathrm{GraB}$ alone. Untreated cells lysed with Triton X-100 were used as a positive control to induce epitope exposure for both molecules. $^{28}$ We therefore concluded that Bak and Bax were maintained in their non-active conformations unless Bcl-2 was neutralised by $\mathrm{ABT}-737$, and that the presence of gctBid must therefore be required to maintain the sensitivity of the GraBtreated cells to ABT-737.

To determine how Bcl-2 was acting, we used co-immunoprecipitation to determine whether gctBid, Bax and/or Bak was directly bound to $\mathrm{Bcl}-2$. Bax did not co-precipitate with Bcl-2 following treatment with Pfp/GraB alone, but did following ABT-737 addition (Figure 4a). Bak did not associate with Bcl-2 either before or after ABT-737 (data not shown), although this may merely reflect the reduced ability of Bak to bind to the $\mathrm{Bcl}-2$ overexpressed in these cells. ${ }^{24,26}$ The observed $\mathrm{Bax} / \mathrm{Bcl}-2$ binding did not prevent translocation of Bax to the mitochondria or prevent MOMP as cyt $c$ was still released in cells treated with $\mathrm{Pfp} / \mathrm{GraB}$ and $\mathrm{ABT}-737$. These data suggest that $\mathrm{Bcl}-2$ does not block $\mathrm{GraB}$ by directly binding Bax (or Bak), and consequently that ABT-737 does not promote MOMP by displacing Bax or Bak from Bcl-2. In contrast, we found that gctBid did co-precipitate with $\mathrm{Bcl}-2$ in cells treated with $\mathrm{Pfp} / \mathrm{GraB}$ alone (Figure $4 \mathrm{~b}$ ), so this binding may prevent gctBid from activating Bax or Bak. Although addition of ABT-737 killed these cells, it did not displace large amounts of gctBid from Bcl-2. Rather ABT-737 generated cctBid, which could also associate with Bcl-2. In dying cells, the overexpressed $\mathrm{Bcl}-2$ could therefore bind gctBid, cctBid, activated Bax and presumably ABT-737, but not sufficiently well to block apoptosis.

Together, the data clearly show that the GraB signal involves direct Bid cleavage. This signal is transduced as far as mitochondria, but is held in check by Bcl-2 upstream of Bax/Bak activation. We note importantly that gctBid (but not cctBid, Bak or Bax) was bound to Bcl-2 before ABT-737 addition. A small amount of displaced gctBid may then activate $\mathrm{Bax} / \mathrm{Bak}$, but this requires confirmation.

GctBid persists in cells during the 'window of opportunity' for sensitivity to ABT-737. For gctBid to maintain the pressure to die in GraB-treated cells, it must a
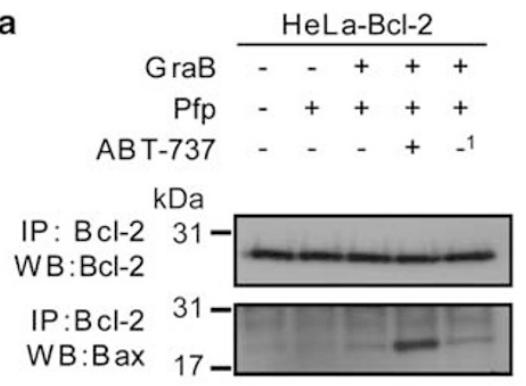

b

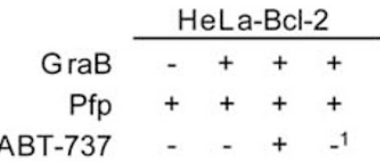

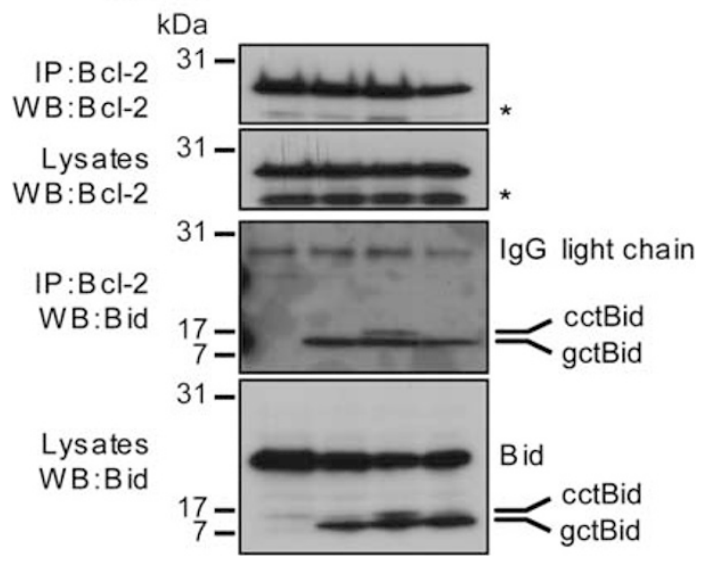

Figure $4 \mathrm{Bcl}-2$ sequesters gctBid in the absence of ABT-737 but only binds $\mathrm{Bax}$ after treatment with ABT-737. HeLa-Bcl-2 cells were treated with Pfp/GraB for $1 \mathrm{~h}$ and a further $30 \mathrm{~min} \pm A B T-737(500 \mathrm{nM})$ before lysis in $1 \%$ digitonin lysis buffer. (a) Bcl-2 was immunoprecipitated and immunoblotted for Bcl-2 (upper panel) or Bax (lower panel). (b) Similarly Bcl-2 was again immunoprecipitated and immunoblotted for Bcl-2 (top panel) or Bid (both the GraB (gc) and caspase (cc) cleaved forms; third panel). Whole-cell lysates were also immunoblotted for Bcl-2 (second panel) and Bid (bottom panel). ${ }^{1}$ After Pfp/GraB treatment cells were incubated for an additional $30 \mathrm{~min}$ in the absence of ABT-737. *Is a non-specific band. The experiment was performed twice

persist throughout the 'window of opportunity' during which GraB-treated cells were sensitive to ABT-737. We therefore determined using immunoblot analysis that gctBid is present after an extended time (17-18h) and confirmed that cyt $c$ was released once ABT-737 was added at this time, even if the GraB is no longer active (Figure 5). Pfp/GraB were added to HeLa and HeLa-Bcl-2 cells $(t=0)$ and caspase activity was completely blocked with the inhibitor Q-VD-OPh. GraB inhibitor, C20, was added to HeLa cells at $t=0 \mathrm{~h}$ to confirm that cleavage of Bid and loss of cyt $c$ were completely blocked in HeLa cells under these conditions. To assess the need for on-going GraB activity in HeLa-Bcl-2 cells, the same concentration of $\mathrm{C} 20$ was added at $t=2 \mathrm{~h}$ to block any further GraB-mediated Bid cleavage and ABT-737 addition was delayed for a further $15 \mathrm{~h}(t=17 \mathrm{~h})$, as indicated. Finally, cells were fractionated $30 \mathrm{~min}$ later and assayed for the presence of gctBid and loss of cyt $c$ from the mitochondrial fraction. Although the level of gctBid detected in the mitochondria was reduced in the $\mathrm{Bcl}-2$ cells in which $\mathrm{C} 20$ was added (top panel, 


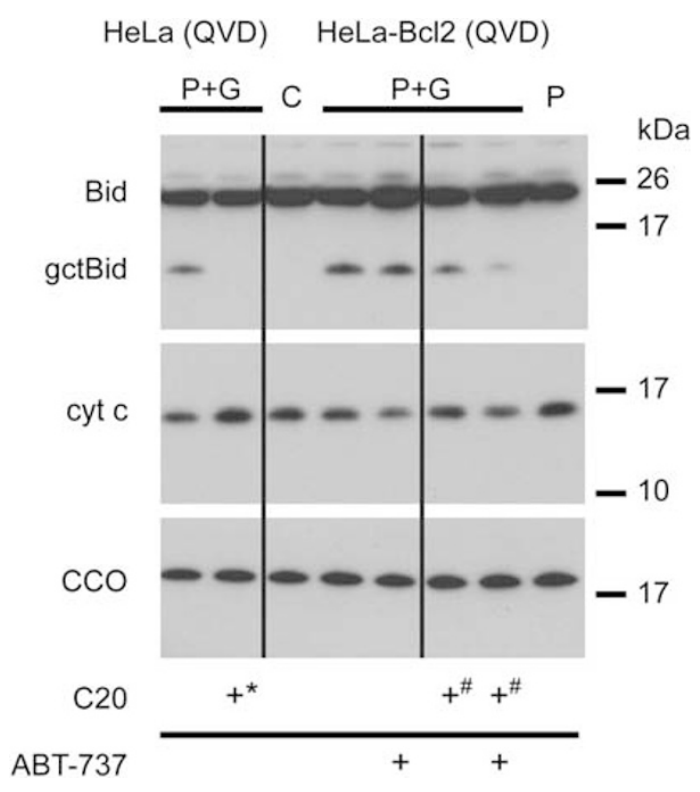

Figure 5 Prolonged maintenance of gctBid is associated with cyt $c$ release after ABT-737 treatment up to $18 \mathrm{~h}$ after initiation of the GraB death signal. HeLa and HeLa-Bcl-2 cells were treated with Pfp/GraB in the presence of Q-VD-OPh (QVD; $20 \mu \mathrm{M})$. C20 $(10 \mu \mathrm{M})$ was either added to HeLa cells (lane marked as C20*) together with the Pfp/GraB (at $0 \mathrm{~h}$ ) or to the Bcl-2 cells after $2 \mathrm{~h}$ (lanes marked as $\left.\mathrm{C} 20^{\#}\right)$. ABT-737 $(500 \mathrm{nM})$ was added to $\mathrm{Bcl}-2$ cells as indicated after a further $15 \mathrm{~h}$ $(t=17 \mathrm{~h})$ and the cells were fractionated at $17.5 \mathrm{~h}$. Mitochondrial fractions were examined by Western blot analysis for the presence of gctBid (upper panel) and cyt $c$ loss from the mitochondria (middle panel). cyt coxidase (CCO) levels indicated equal protein loading (lower panel). Results are representative of three similar experiments

compare lanes 6 and 7 to lanes 4 and 5), the addition of ABT737 still resulted in equivalent loss of cyt $c$, indicating that MOMP had taken place (compare lanes $7-5$, middle panel). Reduced gctBid levels were detected in a similar experiment (Supplementary Figure 3) where the loss of mitochondrial cyt $c$ was also associated with increased cyt $c$ levels in the cytosol.

Overall, we showed that GraB induces a long-lived (up to $20 \mathrm{~h}$, Figure $2 \mathrm{~b}$ and Figure 5) death signal that was held in check by Bcl-2 but could be de-repressed by ABT-737. The presence of gctBid at mitochondria many hours after GraB proteolysis had stopped suggesting that gctBid bound to $\mathrm{Bcl}-2$ is the long-lived signal generated by $\mathrm{GraB}$, which renders $\mathrm{Bcl}-2$ overexpressing cells sensitive to ABT-737. After $24 \mathrm{~h}$, the cells resumed their resistance to either stimulus (Pfp/GraB or ABT-737) alone, but remained sensitive to both stimuli in combination.

\section{Discussion}

ABT-737 is a small molecule antagonist of $\mathrm{Bcl}-2$ being trialled alone or in combination with cytotoxic drugs. ${ }^{29}$ Our previous studies showed that co-overexpression of $\mathrm{Bcl}-2$ and XIAP confer resistance of cancer cells to NK-induced death ${ }^{14}$ and this may explain why some patients do not respond or relapse following therapy. Our current study demonstrates that ABT737 can restore the mitochondrial pathway in the target cells and validates the potential of ABT-737 to be effective in combination with cell-based immunotherapy. Although

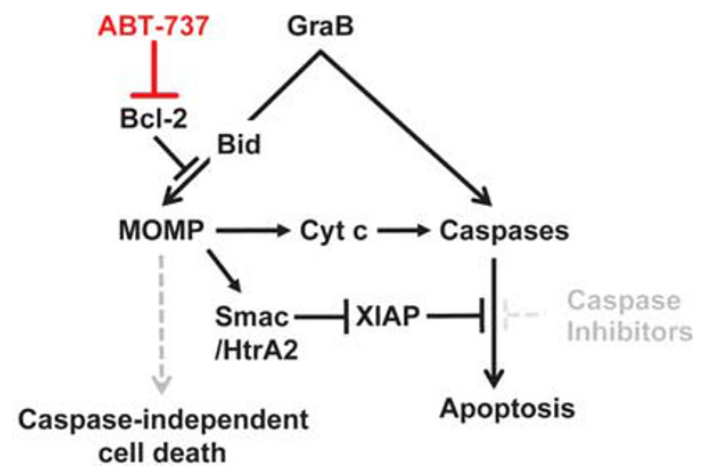

Figure 6 Restoration of cell death by ABT-737 in cancer cells that overexpress $\mathrm{Bcl}-2$ and caspase activity is blocked. GraB can activate a mitochondria-dependent pathway to cell death by cleaving Bid (left), which triggers MOMP. This pathway can be blocked by overexpression of $\mathrm{Bcl}-2$. GraB can also trigger apoptosis by cleaving caspases directly (right) and this pathway can be blocked by overexpression of caspase inhibitors, such as XIAP. Cell death can therefore be achieved by the complementary pathway if only one pathway is blocked. In cases where both pathways are blocked, neutralising Bcl-2 with ABT-737 is sufficient to restore the mitochondrial pathway. This results in apoptosis by activation of caspases, which can be achieved directly via cyt $c$ or indirectly by Smac/HtrA2 that can neutralise some caspase inhibitors. MOMP can also cause caspase-independent cell death as a consequence of mitochondrial damage. Restoration of GraB-induced MOMP by neutralising Bcl-2 will therefore cause death of the cell even if caspase activity cannot be restored

ABT-737 can only restore the mitochondria-dependent pathway, MOMP also causes release of Smac/DIABLO to overcome $\mathrm{XIAP}$. ABT-737 is therefore likely to restore NK-induced apoptosis in cancer cells that co-overexpress Bcl-2 and XIAP (Figure 6). Just as some viruses express caspase inhibitors that are not neutralised by Smac/DIABLO (e.g., CrmA), some cancer cells harbour defects that prevent them from executing mitochondria-independent pathways to apoptosis (e.g., MCF-7 breast cancer cells are deficient in caspase-3). ${ }^{30}$ ABT-737 may not fully restore NK-induced apoptosis in these cells but Bax/Bak-mediated mitochondrial damage could still induce caspase-independent death. We therefore suggest that ABT-737 may be useful together with immunotherapy using CL to kill cancer cells, even those that harbour defects in caspase activation.

We showed that the pro-apoptotic signal generated by GraB is surprisingly long lived (up to $20 \mathrm{~h}$ ) and that this signal depended on persistent gctBid even when GraB proteolytic activity had long been inhibited (Figure 5, Supplementary Figure 3). Importantly this suggests that timing of ABT-737 administration might be critical for the success of CTL/NK therapies. For example, delayed treatment with ABT-737, possibly $12-16 \mathrm{~h}$ after delivery of the NK cells, is likely to generate a large pool of cancer cells 'primed' for death and therefore sensitive to ABT-737. Further, NK can persist for many days in the tumour environment and a single killer cell can encounter multiple targets in succession. Therefore, sequential doses of ABT-737 12-16 h apart may be more effective than a single-dose delivered at the same time as immunotherapy. We also showed that target cells that survive ABT-737 following the initial treatment with $\mathrm{Pfp} / \mathrm{GraB}$ are still sensitive to subsequent treatments with $\mathrm{Pfp} / \mathrm{GraB}$ and $A B T-737$ (Figure 2c). Strategies that prolong the life of gctBid may also be worth exploring as degradation of 
tBid is a control element in many apoptotic pathways and stabilisation of tBid, or proteosome inhibition can greatly amplify apoptosis. ${ }^{31}$

In addition to understanding the therapeutic potential of ABT-737 during cell-based immunotherapy our study elucidates how ABT-737 synergises with GraB to restore the mitochondrial death pathway. We found that gctBid translocated to mitochondria, but is bound to and held in check by $\mathrm{Bcl}-2$ upstream of Bax/Bak activation. This block was rapidly neutralised by $\mathrm{ABT}-737$ resulting in MOMP within $15 \mathrm{~min}$. Interestingly, substantial amounts of gctBid remained bound to $\mathrm{Bcl}-2$ after addition of ABT-737 even when cells were clearly destined to die. However, our data suggest that only partial release of gctBid (undetectable by immunoblot) from $\mathrm{Bcl}-2$ may be required to activate Bax/Bak, a rapid and stochastic process that has been notoriously difficult to monitor, and the subject of intense study. ${ }^{32}$ It is therefore likely that gctBid reduces the capacity of $\mathrm{Bcl}-2$ to retain cell viability, as has been shown for $\operatorname{cctBid}^{33,34}$ and that Bax and Bak only become involved downstream of the gctBid/Bcl-2 interaction.

Our findings are consistent with a previous study that used plasmid transfection to deliver different levels of tBid, which mimicked cctBid. Cells primed to die with lower levels of tBid underwent MOMP faster $(0-20 \mathrm{~min})$ than cells with higher levels of $t$ Bid (40-120 min) following ABT-737 addition. ${ }^{27}$ This model predicted that Bcl-2 blocked lower levels of tBid by a mechanism designated mode 1 , but higher levels of tBid activated Bax and Bak, blocked by a different mechanism designated mode 2. Bcl-2 appears to block GraB-induced death by mode 1 in our model (direct antagonism of gctBid) as the signal was rapidly released by ABT-737 ( $<20 \mathrm{~min}$ ) and did not involve prior activation of Bax or Bak. This questions whether mode 2 inhibition can be achieved when Bid is activated under physiological conditions. Our model, which is more physiological and therapeutically relevant, should be useful for future studies aiming to unravel the complex sets of interactions between pro- and anti-apoptotic Bcl-2 family members ultimately leading to MOMP, and thereby influence drug design to restore the mitochondrial pathway in cancer cells where MOMP is blocked.

\section{Materials and Methods \\ Cell culture and reagents. HeLa cells transfected with cyt c-GFP (clone $2 \mathrm{H} 18$ ) and also transfected with human $\mathrm{Bcl}-2^{9}$ were maintained in DMEM (supplemented with $2 \mathrm{mM}$ glutamine and $10 \%$ FBS). Human NK cells were generated as described previously and maintained in supplemented RPMI-1640 containing rhlL-2. ${ }^{14}$ Caspases in target cells were blocked using either ZVAD-fmk $(100 \mu \mathrm{M}$, Enzyme Systems Products, Dublin, CA, USA) or Q-VD-OPh $(20 \mu \mathrm{M}$, Calbiochem, La Jolla, CA USA). Human GraB was inhibited by either pre-incubation of target cells with C20 $(10 \mu \mathrm{M}$; SYN/THESIS Med Chem, Shanghai, China) $)^{14}$ for $30 \mathrm{~min}$ at $37^{\circ} \mathrm{C}$ or the addition of the inhibitor after treatment of cells with Pfp/GraB. The Bcl-2 inhibitor ABT-737, or its enantiomer (Abbott Laboratories, Abbott Park, IL, USA) were added to cells at a final concentration of 250-500 nM. Recombinant GraB was produced in Pichia pastoris ${ }^{35}$ and recombinant mouse Pfp was produced in a baculovirus expression system. $^{14}$}

Cell death assays. Cell death induced by GraB (12.5-25nM) and Pfp ( $1 \mathrm{nM})$ was quantitated by chromium $\left({ }^{51} \mathrm{Cr}\right)$ release, annexin $\mathrm{V}$ binding and cyt $c$ release as previously described. ${ }^{13,14,18}$ Death of target cells induced by human NK was assessed by release of cyt $c$. Cells were fixed and stained using a specific antibody as previously described. ${ }^{14}$
Immunocytochemistry. The translocation of Bax from the cytosol to the mitochondria and the release of cyt $c$ from the mitochondria to the cytosol was determined by immunocytochemistry, as described previously, ${ }^{14}$ using antibodies specific for either cyt $c$ (clone 6H2.B4) or Bax (6A7) followed by an anti-mouse Alexa 594 secondary antibody (Life Technologies, Mulgrave, VIC, Australia). Cells were plated overnight in 8-well chamber slides before treatment with GraB $(12.5 \mathrm{nM})$, Pfp $(18 \mathrm{nM})$, zVAD $(100 \mu \mathrm{M})$ and ABT-737 (500nM). Cells were visualised on an Olympus FV1000 confocal microscope (Mount Waverley, VIC, Australia).

FACS. Cells with active Bax were detected by flow cytometry (BD Biosciences, BD FACS Canto II, Franklin Lakes, NJ, USA), using an antibody, specific for the conformation change (6A7). The antibody was used on fixed and permeabilised cells, as described previously, ${ }^{36}$ at a $1: 100$ dilution and detected with an anti-mouse Alexa 594 secondary antibody.

Western blot analysis. Typically $1-2 \times 10^{6}$ cells were treated with 7.5-15 $\mu \mathrm{M} \mathrm{GraB}$ and 2.2 nM Pfp for 45-60 min. ABT-737 (500 nM) (20-30 min, $37^{\circ} \mathrm{C}$ ) was added as indicated. Whole-cell lysates (in $0.1 \%$ NP40) were separated on either 12 or $15 \%$ acrylamide gels and probed using specific monoclonal antibodies to caspase-3 (BD Transduction Laboratories, San Jose, CA, USA) caspase-7 and caspase-9 (BD Pharmingen, San Diego, CA, USA), and Bid (clone no. $2 \mathrm{D} 1)^{37}$ as previously described. ${ }^{7}$

Immunoprecipitation. Following treatment with Pfp/GraB and ABT-737 as described above, cells were lysed in lysis buffer $(20 \mathrm{mM}$ Tris- $\mathrm{HCl}$ pH 7.4, $135 \mathrm{mM}$ $\mathrm{NaCl}, 1.5 \mathrm{mM} \mathrm{MgCl} 2,1 \mathrm{mM} \mathrm{EGTA}, 10 \%$ glycerol) containing $1 \%$ digitonin or $1 \%$ TX-100 supplemented with Complete protease inhibitors (Roche, Basel, Switzerland) for $30 \mathrm{~min}$ on ice. Insoluble debris was pelleted and lysates were pre-cleared with a $1: 1$ slurry of sepharose in lysis buffer for $1 \mathrm{~h}$ at $4^{\circ} \mathrm{C}$ with constant agitation. Immunoprecipitation was performed for $2 \mathrm{~h}$ at $4{ }^{\circ} \mathrm{C}$ with antiBcl-2 (Bcl-2-100, BD Biosciences), anti-Bax (6A7, BD Biosciences) or anti-Bak (Ab-1, Calbiochem). Immune complexes were captured with Protein G/sepharose for $1 \mathrm{~h}$ at $4{ }^{\circ} \mathrm{C}$, washed four times with lysis buffer and eluted by boiling in SDSPAGE sample buffer. Immunoprecipitates and lysates were run on SDS-PAGE and immunoblotted for Bcl-2 (clone no. 7, BD Biosciences), Bax (N-20, Santa Cruz Biotechnologies, Santa Cruz, CA, USA), Bak (B5929, Sigma-Aldrich, Castle Hill, New South Wales, Australia), or Bid (clone no. 2D1). ${ }^{37}$

Subcellular fractionation. To investigate the cellular location of Bax and Bak, cells were harvested and resuspended in permeabilization buffer $(20 \mathrm{mM}$ HEPES/KOH pH 7.5, $100 \mathrm{mM}$ sucrose, $2.5 \mathrm{mM} \mathrm{MgCl}, 50 \mathrm{mM} \mathrm{KCl}, 0.025 \%$ digitonin and complete protease inhibitors (Roche Applied Science, Castle Hill, New South Wales, Australia) for $10 \mathrm{~min}$ on ice. Permeabilized cells were centrifuged at $13000 \mathrm{~g}$ for $5 \mathrm{~min}$ at $4^{\circ} \mathrm{C}$ and the supernatant (cytosol, C) and pellet (membranes, M) were separated and boiled in reducing SDS-PAGE sample buffer. Fractions were run on SDS-PAGE and immunoblotted for Bax (N-20, Santa Cruz Biotechnologies) or Bak (B5929, Sigma, St. Louis, MO, USA). The cellular location of tBid and cyt $c$ following Pfp/GraB, with and without ABT-737 was also determined by western blot analysis using antibodies to Bid (clone no. 2D1) and cyt $c$ (clone $7 \mathrm{H} 8.2 \mathrm{C} 12)$. Cells were fractionated into membrane and cytosolic fractions, as previously described. ${ }^{15}$ Equal loading was assessed using antibodies to either $\beta$-actin (cytosolic) (AC-74, Sigma-Aldrich) or bovine cyt $c$ oxidase, subunit IV (mitochondrial) (Molecular Probes, Leiden, The Netherlands).

\section{Conflict of Interest}

The authors declare no conflict of interest.

Acknowledgements. This work was supported by funding from the NHMRC (Project Grant and CDA Award to NJW, project grants \#575559 and \#637335 to RMK, Programme Grant to JAT, RJW and PB), the Australian Research Council (Futures fellowship to NJW), project grants from the Leukaemia Foundation Queensland and the Prostate Cancer Foundation Australia to NJW. We thank Abbot Laboratories for ABT-737, Ms Kate Whitecross, Dr. Jamie Lopez and Dr. Daniella Brasacchio for helpful technical discussions, Ms Diana Motion for editorial assistance, and Mr Kevin Thia for graphical expertise. Owing to editorial limits we were unable to cite all relevant publications. 
1. Waterhouse NJ, Trapani JA. CTL: Caspases Terminate Life, but that's not the whole story. Tissue Antigens 2002; 59: 175-183.

2. Trapani JA, Sutton VR. Granzyme B: pro-apoptotic, antiviral and antitumor functions. Curr Opin Immunol 2003; 15: 533-543.

3. Zitvogel L, Tesniere A, Apetoh L, Ghiringhelli F, Kroemer G. Immunological aspects of cancer chemotherapy. Nat Rev Immunol 2008; 8: 59-73.

4. Froelich CJ, Orth K, Turbov J, Seth P, Gottlieb R, Babior B et al. New paradigm for lymphocyte granule-mediated cytotoxicity. Target cells bind and internalize granzyme $B$ but an endosomolytic agent is necessary for cytosolic delivery and subsequent apoptosis. J Biol Chem 1996; 271: 29073-29079.

5. Shi L, Mai S, Israels S, Browne K, Trapani JA, Greenberg AH. Granzyme B (GraB) autonomously crosses the cell membrane and perforin initiates apoptosis and $\mathrm{GraB}$ nuclear localization. J Exp Med 1997; 185: 855-866.

6. Kaiserman D, Bird CH, Sun J, Matthews A, Ung K, Whisstock JC et al. The major human and mouse granzymes are structurally and functionally divergent. J Cell Biol 2006; 175: 619-630.

7. Sutton VR, Davis JE, Cancilla M, Johnstone RW, Ruefli AA, Sedelies K et al. Initiation of apoptosis by granzyme $B$ requires direct cleavage of bid, but not direct granzyme B-mediated caspase activation. J Exp Med 2000; 192: 1403-1414

8. Barry M, Heibein JA, Pinkoski MJ, Lee SF, Moyer RW, Green DR et al. Granzyme B short-circuits the need for caspase 8 activity during granule-mediated cytotoxic T-lymphocyte killing by directly cleaving Bid. Mol Cell Biol 2000; 20: 3781-3794.

9. Pinkoski MJ, Waterhouse NJ, Heibein JA, Wolf BB, Kuwana T, Goldstein JC et al. Granzyme B-mediated apoptosis proceeds predominantly through a Bcl-2-inhibitable mitochondrial pathway. J Biol Chem 2001; 276: 12060-12067.

10. Alimonti JB, Shi L, Baijal PK, Greenberg AH. Granzyme B induces BID-mediated cytochrome $C$ release and mitochondrial permeability transitionJ Biol Chem 2001; 276: 6974-6982.

11. Luo X, Budihardjo I, Zou H, Slaughter C, Wang X. Bid, a Bcl2 interacting protein, mediates cytochrome $c$ release from mitochondria in response to activation of cell surface death receptors. Cell 1998; 94: 481-490.

12. Kluck RM, Bossy-Wetzel E, Green DR, Newmeyer DD. The release of cytochrome c from mitochondria: a primary site for Bcl-2 regulation of apoptosis. Science 1997; 275: 1132-1136.

13. Sutton VR, Vaux DL, Trapani JA. Bcl-2 prevents apoptosis induced by perforin and granzyme B, but not that mediated by whole cytotoxic lymphocytes. J Immunol 1997; 158: 5783-5790

14. Sedelies KA, Ciccone A, Clarke CJ, Oliaro J, Sutton VR, Scott FL et al. Blocking granule-mediated death by primary human NK cells requires both protection of mitochondria and inhibition of caspase activity. Cell Death Differ 2008; 15: 708-717.

15. Sutton VR, Wowk ME, Cancilla M, Trapani JA. Caspase activation by granzyme B is indirect, and caspase autoprocessing requires the release of proapoptotic mitochondrial factors. Immunity 2003; 18: 319-329.

16. Lickliter JD, Cox J, McCarron J, Martinez NR, Schmidt CW, Lin H et al. Small-molecule $\mathrm{Bcl}-2$ inhibitors sensitise tumour cells to immune-mediated destruction. Br J Cancer 2007; 96: 600-608.

17. Waterhouse NJ, Goldstein JC, von Ahsen O, Schuler M, Newmeyer DD, Green DR. Cytochrome $\mathrm{c}$ maintains mitochondrial transmembrane potential and ATP generation after outer mitochondrial membrane permeabilization during the apoptotic process. $J$ Cell Bio 2001; 153: 319-328.

18. Waterhouse NJ, Sedelies KA, Sutton VR, Pinkoski MJ, Thia KY, Johnstone R et al Functional dissociation of DeltaPsim and cytochrome $\mathrm{c}$ release defines the contribution of mitochondria upstream of caspase activation during granzyme B-induced apoptosis. Cell Death Differ 2006; 13: 607-618.

19. Kluck RM, Esposti MD, Perkins G, Renken C, Kuwana T, Bossy-Wetzel E et al. The pro-apoptotic proteins, Bid and Bax, cause a limited permeabilization of the mitochondrial outer membrane that is enhanced by cytosol. J Cell Biol 1999; 147: 809-822.

20. von Ahsen O, Renken C, Perkins G, Kluck RM, Bossy-Wetzel E, Newmeyer DD. Preservation of mitochondrial structure and function after Bid- or Bax-mediated cytochrome c release. J Cell Biol 2000; 150: 1027-1036.
21. Desagher S, Osen-Sand A, Nichols A, Eskes R, Montessuit S, Lauper S et al. Bid-induced conformational change of $\mathrm{Bax}$ is responsible for mitochondrial cytochrome $\mathrm{c}$ release during apoptosis. J Cell Biol 1999; 144: 891-901.

22. Wei MC, Zong WX, Cheng EH, Lindsten T, Panoutsakopoulou V, Ross AJ et al. Proapoptotic BAX and BAK: a requisite gateway to mitochondrial dysfunction and death. Science 2001; 292: 727-730.

23. Cheng EH, Wei MC, Weiler S, Flavell RA, Mak TW, Lindsten T, Korsmeyer SJ. BCL-2, $\mathrm{BCL}-\mathrm{X}(\mathrm{L})$ sequester $\mathrm{BH} 3$ domain-only molecules preventing BAX- and BAK-mediated mitochondrial apoptosis. Mol Cell 2001; 8: 705-711.

24. Willis SN, Chen L, Dewson G, Wei A, Naik E, Fletcher JI, Adams JM. Proapoptotic Bak is sequestered by $\mathrm{Mcl}-1$ and $\mathrm{Bcl}-\mathrm{xL}$, but not $\mathrm{Bcl}-2$, until displaced by $\mathrm{BH} 3-$ only proteins. Genes Dev 2005; 19: 1294-1305.

25. Ding J, Zhang Z, Roberts GJ, Falcone M, Miao Y, Shao Y et al. Bcl-2 and Bax interact via the $\mathrm{BH} 1-3$ groove- $\mathrm{BH} 3$ motif interface and a novel interface involving the $\mathrm{BH} 4$ motif. J Biol Chem 2010; 285: 28749-28763.

26. Dai H, Meng XW, Lee SH, Schneider PA, Kaufmann SH. Context-dependent Bcl-2/Bak interactions regulate lymphoid cell apoptosis. J Biol Chem 2009; 284: 18311-18322.

27. Llambi F, Moldoveanu T, Tait SW, Bouchier-Hayes L, Temirov J, McCormick LL et al. A unified model of mammalian BCL-2 protein family interactions at the mitochondria. $\mathrm{Mol}$ Cell 2011; 44: 517-531.

28. Dewson G, Kratina T, Sim HW, Puthalakath H, Adams JM, Colman PM et al. To trigger apoptosis, Bak exposes its $\mathrm{BH} 3$ domain and homodimerizes via $\mathrm{BH} 3$ :groove interactions. Mol Cell 2008; 30: 369-380.

29. Yecies D, Carlson NE, Deng J, Letai A. Acquired resistance to ABT-737 in lymphoma cells that up-regulate MCL-1 and BFL-1. Blood 2010; 115: 3304-3013.

30. Metkar SS, Wang B, Ebbs ML, Kim JH, Lee YJ, Raja SM et al. Granzyme B activates procaspase-3 which signals a mitochondrial amplification loop for maximal apoptosis. $J$ Cell Biol 2003; 160: 875-885.

31. Breitschopf K, Zeiher AM, Dimmeler S. Ubiquitin-mediated degradation of the proapoptotic active form of bid. A functional consequence on apoptosis induction. J Biol Chem 2000; 275: 21648-21652

32. Youle RJ, Strasser A. The BCL-2 protein family: opposing activities that mediate cell death. Nat Rev Mol Cell Biol 2008; 9: 47-59.

33. Peng J, Tan C, Roberts GJ, Nikolaeva O, Zhang Z, Lapolla SM et al. tBid elicits a conformational alteration in membrane-bound $\mathrm{Bcl}-2$ such that it inhibits Bax pore formation. J Biol Chem 2006; 281: 35802-35811.

34. Willis SN, Fletcher JI, Kaufmann T, van Delft MF, Chen L, Czabotar PE et al. Apoptosis initiated when BH3 ligands engage multiple Bcl-2 homologs, not Bax or Bak. Science 2007; 315: 856-859.

35. Sun J, Bird CH, Buzza MS, McKee KE, Whisstock JC, Bird PI et al. Expression and purification of recombinant human granzyme B from Pichia pastoris. Biochem Biophys Res Commun 1999; 261: 251-255.

36. Bird $\mathrm{CH}$, Sutton VR, Sun J, Hirst CE, Novak A, Kumar $\mathrm{S}$ et al. Selective regulation of apoptosis: the cytotoxic lymphocyte serpin proteinase inhibitor 9 protects against granzyme B-mediated apoptosis without perturbing the Fas cell death pathway. Mol Cell Biol 1998; 18: $6387-6398$.

37. Kaufmann T, Tai L, Ekert PG, Huang DC, Norris F, Lindemann RK, Johnstone RW et al. The BH3-only protein bid is dispensable for DNA damage- and replicative stress-induced apoptosis or cell-cycle arrest. Cell 2007; 129: 423-433.

Cell Death and Disease is an open-access journal published by Nature Publishing Group. This work is licensed under the Creative Commons Attribution-NonCommercial-No Derivative Works 3.0 Unported License. To view a copy of this license, visit http://creativecommons.org/licenses/by-nc-nd/3.0/

\section{Supplementary Information accompanies the paper on Cell Death and Disease website (http://www.nature.com/cddis)}

